\title{
Origins and Mechanisms in the Development of Major Mental Disorders: A Clinical Approach
}

\author{
Nicholas Pediaditakis \\ American Psychiatric Association, Arlington, USA \\ Email: Niko15@bellsouth.net
}

Received October 15, 2011; revised November 7, 2011; accepted December 5, 2011

\begin{abstract}
This paper considers the following collective significance of the shared, clinical characteristics of the major mental disorders (MMDs), their co-morbidities, overlaps and pharmacological responses with the following conclusions: 1) These disorders have a common, initial, neurodevelopmental origin. 2) They can occur probabilistically on susceptible individuals, on account of pre-existing, extreme, temperamental variances-signifying underlying structural variance. 3) Each of these syndromes can be considered the expression of disturbances in the overall, common, operating mode of brain function which normally ensures the synchrony, coordination, elegance and subtlety in the expression of all the brain's higher faculties. 4) Lastly, this function is a complex, emergent phenomenon based on the individual's temperamental/structural underlying makeup, switching intermittently from a normal phase to a pathologically, ordered one-the latter phase expressing itself with symptoms made up of expressing either/or, antithetical substitutes for each of the MMDs-similar to the fluctuations found in patient's with Parkinson's disease.
\end{abstract}

Keywords: Temperament; Major Mental Disorders; Comorbidity; Symptomatology; Oscillations; Synchrony; Operating Mode

\section{Introduction}

Empirical clinical evidence and previous research demonstrate shared characteristics that together play crucial roles in understanding the development of MMDs. These characteristics, although readily apparent clinically are not fully appreciated in the context of their collective significance in the underlying causes of the MMDs. This paper reviews several classic shared characteristics and discusses a novel perspective on their collective significance leading to diverse pathologies. These clinical phenomena and their collective significance have being overlooked perhaps due to the procrustean guidelines of the DSM-IV (e.g., essentially a Krepelinian, nosological schema), acting as a semantic and conceptual trap as well as for practical consideration in billing. The clinician also accommodates the clinical dissonance observed in a patient by using terms like "co-morbidity", thus avoiding a new clinical picture whereby the same individual may actually have a new, more complex manifestation of the same underlying disease. In addition, we use terms like "non elsewhere classified" in ill-fitting, clinical syndromes. The conclusions in this paper have important ramifications for reconsidering the origin and development of the MMDs for future research and the develop- ment of novel, less intrusive types of treatment.

\section{The Significance of Inborn Propensities (Temperament)}

Temperament is an important but grossly understudied (understandable due to the difficulties in studying it) phenomenon. It can be defined as the inborn "How" of behavior and is made up partially and somewhat controversially of the following elements [1-3]: 1) emotionality, 2) intensity versus reactivity, 3) cerebricity (i.e., thinking the world versus feeling the world), 4) sociability, 5) aloofness versus detachment, 6) compulsivity (algorithmic faculty), and 7) inner versus other directedness.

These elements, as well as perhaps others, tend to occur in clusters in a variety of distinct ways combining and forming different types of temperament [4-6]. They constitute a scaffold on which a person's particular personality is built. Environmental influences affect the temperamental components- the later tend to recruit the type and significance of early events that shape such temperament-adding lifelong attitudes and habits as well as early imprints whether faulty or not. Eventually, such mix of inborn temperament and environmental factors comprise the totality of an individual's personality.

Temperament, in addition to its role in MMDs, ap- 
pears to be significant in other aspects of an individual's life. It is partially involved in mate selection for the formation of procreational dyads-choosing one's mate complementarily of each other's temperaments-contributing along with other factors, to the longevity of such dyad, necessary for maturation of the human baby, as well as perpetuating lasting partnerships [7]. It also contributes to one's lifestyle and vocational choice within the framework of contingency and circumstance. It also acts as a facilitator in the presence of certain extreme components (aloofness/inner directedness), as in the case of gifted and talented individuals. It helps to conduit their abilities into creative channels by mechanisms not yet clear [810]. Evolutionarily, temperament may also contribute specific benefits to a group as a whole, not just to the individual.

The underlying temperamental variances in all MMDs should be viewed as extreme degrees in the expression of normally occurring types. Normal temperamental components do not impair relationships and actions, nor create discomfort or anguish under normal circumstances. They represent inborn behavioral tendencies expressing variable styles for coping and survival, while often also contributing to the survival of an individual's tribe. They represent underlying structural variances in the very architecture of the brain [11].

In the case of schizophrenia, preexisting extreme temperamental variances before the onset of a disorder make up the pre-morbid personality [12]. At the onset and relapse, the specific extreme temperamental elements are considered part of the disorder and now called negative symptoms even though were part one's temperament all along - as the patient himself and relatives ascertain if asked [13]. In contrast, the appearance of the periodic psychoses heralding the onset and naming of the disorder are called the positive symptoms. Using different terms for a clinical phenomenon during different times act as a "semantic trap". Actually, the positive psychotic symptoms should be viewed as periodic epiphenomena on preexisting, extreme temperamental variances [14].

On the other hand, preexisting specific temperamental variances in other MMDs are not usually considered causative factors in a disorder. Usually, with rare exceptions, they are ignored as to their causative role or merely labeled as traits $[15,16]$. Extreme temperamental variances for each disorder remain unaffected in remissions and, specifically for schizophrenia, are now called "residual symptoms". They are often mixed with some lingering positive ones [17-19].

It appears that individuals with extreme temperamental variances constitute a pool of vulnerable candidates. Some of these candidates will subsequently and probabilisticcally develop a MMD usually in late adolescence, presumably under social and hormonal pressures as well as having other possible elements (e.g., pruning) acting as precipitating factors.

Specifically in schizophrenia, the preexisting, extreme temperamental variances that occur in various combinations include: 1) aloofness, 2) apathy punctuated by occasional anger tantrums, 3) social uneasiness, 4) self-absorption, 5) absence of empathy, 6) inattentiveness/indifference and 7) an inability to accommodate ambiguity.

In individuals afflicted with obsessive-compulsive disorder (OCD), there preexists specific extreme temperamental variances' including intolerance of ambiguity. The sufferers tend to be captive to algorithms and express a mirthless attachment to exactitudes as they perform their tasks [17]. It is important to note in the case of OCD that the emergence of disorder may take place irrespective of preexisting temperamental extreme variants in situations where brain functions are threatened by perceived dangers from within or without. It can be considered as an emergency response for constructing a sense of order. Examples include post brain trauma sequelae, prodromal symptoms of impending psychoses, or an exaggerated response to severe external pressures [16]. Manifestations are similar to those of the hero in the film, The Bridge Over River Kwai, or the abused wife who compulsively ended up eating raw sardines in the movie The Tin Drum.

In bipolar mood disorders (BMDs) the preexisting extreme temperamental variances include the following: 1 ) undue emotionality, 2) an acute sensitivity to even mild social stimuli, 3) obsessive behavior, 4) and emotional entrainment. (e.g., a patient's "inner, frozen, landscape", "emotional shackles" or sense of "woodenness", as an afflicted individual will explain in response to inquiry) [20].

In borderline disorder-a misnomer, i.e., "borderline to what?-this variant triggers frequent, spectacular but brief oscillatory symptoms across all the higher mental faculties includes hyper-intensity/reactivity- or a better term "Disorganizing hyper-intensity disorder". Additional temperamental variances for this disorder are lack of empathy and self-absorption [20].

In phobias, anxieties, and panic attacks, the extreme temperamental variances may include the presence of excessive sociability and obedience to social gestalt, excessive empathy, and sensitivity to social expectations and stimuli [20].

In conclusion, all MMDs preexisting temperamental elements-often overlapping-antedating a disorder's "psychotic" phase suggest a common underlying vulnerability, or sensitivity to initial condition [21].

\section{The Significance of Comorbidity and Overlaps during the Expression of the MMDs}

In all MMDs, comorbidity is ubiquitous [22-25]. In addi- 
tion, the syndromes, rarely if ever conform to the procrustean categorical guidelines of the current nosological schema of the Diagnostic and Statistical Manual IV and its international nosological version ICD-10. MMDs. They more often coexist or partially overlap. As G. Claridge aptly states: "Practical manuals are based on categorical divisions of MMDs that may or may not be valid and merely reflect the latest zeitgeist [26].” The psychoses are especially problematic in this respect, because of difficulties in defining their exact boundaries. This leaves considerable room for disagreement and debate, which the ICD and DSM do not address [26]. Further the occurrences of various MMDs is not an either/or phenomenon but they span a range from mild to very severe.

DSM-IV, practical as it is for billing purposes often does not reflect clinical reality. It resembles the old Ptolemaic schema for planetary motions in that it violates the simplicity principle of Occam's Razor. For example, diagnoses were classified as early as the mid 1800's by B. G. Eschenburg as the "usual scheme of the five types of illnesses" using terminology such as "raving madness", "melancholia", "severe foolishness/craziness", "derangement" and "imbecility" [27]. In addition, DSM-IV's procrustean guidelines may be the source of serious flaws in research designs and outcomes. For example, it has been proven statistically in some instances that axis II disorders such as schizophrenia may also be linked to severe forms of affective disorder [28]. It was developed to be descriptive and neutral as to the causes of MMDs. Treating MMDs as if they are distinct, separate entities prevents us from discerning the significance of these phenomena [29-32]. Actually, the existence of unitary psychoses or Einheitpsychose antedates Bleuer's and Kreppelin's constructs from which the current DSM-IV originates. In practice, we accommodate this messiness by using terms like "schizoaffective disorder", "mixed", "atypical psychoses”, "schizotypical”, or "non-else-whereclassified”.

In accordance with the proposed conclusions of this paper and more in accord with the clinical realities, a suggested schema avoiding the pitfalls for research can be mental disorders of the following: 1) mood modulation, 2) thinking, social responses and coordination of feeing with thinking, 3) algorithmic faculty (OCDS), 4) perception of danger from within (anxiety) and without (fear), 5) hyper-intensity as in borderline disorder, and 6) mixed type. In addition, MMDs express themselves in various degrees of severity.

\section{The Significance of Sharing a Family Genetic Pool}

Among family members of patients with MMDs, there exists at a higher rate (16\%) than among the general population (roughly 3\%) of similar or different MMDs as well as extreme temperamental variances [31]. In identical schizophrenic twins, the rate is approximately $50 \%$ for their co-twins [32-35]. This suggests a common initial developmental origin.

\section{The Significance of Periodic Relapses, Remissions and Clinical Shifts}

Given time, all MMDs, regardless of treatment, at least in the initial period will remit and relapse, while underlying extreme temperamental traits remain the same. During relapses, clinical symptoms often shift from one typical syndrome to another, requiring a new diagnosis [34]. Ironically, mental health professionals often assume errors by previous diagnosticians, in an effort to conform to the categorical guidelines.

These phenomena suggest a potential bi-modality-a switch from the normal phase versus an expression of an "either-or", crude, antithetical substitutes, characteristic of the symptomatology found in MMDs.

\section{The Role of Sharing Responses in the Administration of Psychopharmacological Agents and ECT}

Currently available psychotherapeutic agents are moderately effective across the spectrum of all MMDs. They target a single symptom or even symptoms bridging across syndromes irrespective of the named syndrome. This is due to the fact that they usually target particular neurotransmitters $[35,36]$. But current widely used polypharmacy (i.e., the concomitant use of multiple agents each targeting different neurotransmitters affecting the same symptoms) has often better results.

These phenomena suggest that the overall operating mode of brain function is sustained by complex and subtle interplay of many kinds of neurotransmitters as well as the multifunctional role of single neurotransmitter. They also suggest that the overall operating mode is an emergent phenomenon of the prevailing complexity. This is further suggested by delays in the appearance of therapeutic results $[37,38]$.

Clozapine, a dramatically effective drug across syndromes (but unfortunately a dangerous agent due to its side effects) affects several different neurotransmitters [39]. This too suggests a common developmental origin as well as a common overall operating mode of brain function. Other examples such as ECT, Metrazole and old-fashion insulin convulsive therapies affect temporal remissions across the entire spectrum of MMDs by their off-and-on disruptions of brain functions, restoring for a time the normal mode-A kind of rebooting of the operating mode of brain function [40]. 


\section{The Significance of the Overall Operating Mode of Brain Function in MMDs}

The functioning of the normal brain through an overall faculty, ensures a subtlety and synchrony smoothness of actions that determine the elegance and grace in the expression of all higher mental faculties. During the onset and relapse of MMDs, this elegance and subtlety are replaced by a pathologically ordered system characterized by "either-or" misalignments by the brain's neural expression. These pervasive, "either-or" substitutes can be considered as a form of "Psychic-Parkinsonism". Detailed examples of such substitutes have been enumerated elsewhere and a few examples are presented here [1,37]: 1) In the faculty of mood modulation, one's mood oscillates from one extreme to the other, replacing normally amalgamated states of zest moderated by elements of sobriety, constraint, delicacy and subtly. 2) In occurrences of mixed bipolar disorder, an individual's severe anguish and sadness coexists with incongruous joviality and mirth-actually as a rapid oscillation. 3) In the faculty of orderly thinking and the alignment of thinking with sentiments and actions during schizophrenia, one's orderly thinking breaks down into separate, unamalgamated components and are expressed concomitantly in the form of schizophrenic ambivalence-e.g., rapidly oscillating sentiments toward the same person. In addition, with the emergence of the positive symptoms of thought disorder, the functions of orderly thinking, alignment of thinking, appropriate sentiments, and appropriate actions become now uncoordinated.

The very word schizophrenia ( $G k$, "torn-brain function") graphically implies the appearance of odd, and unpredictable non-sequiturs. The occurrences of hallucinations and delusions may plausibly result from decoupling of one's internal normal dialogues with oneself (i.e., reflective thinking and contemplation). The internal dialogues are now perceived by the patient as "coming from external sources", not the patient himself, and are identified as "voices" [39].

Similarly, in delusions, usually persecutory, it may be that the brain attempts to construct a coherent, concrete explanation of the perceived, self-monitored danger to itself during the pathological phase of its overall operating mode. Delusions of grandeur may be an attempt to coherently explain intensely felt, unwarranted emotional exuberance.

The antithetical substitutes phenomena can be best witnessed in their startling form when borderline disorder flares up. The patient oscillates in behavior, expressed feelings, and attitudes within minutes, often in the presence of the examining clinician [2]. For example, a young female sufferer may present herself as a ferocious vixen and then quickly morph into a helpless waif.
In obsessive-compulsive disorders the normal, algorithmic faculty of the brain for fore-planning, sequencing, scheduling the future intended actions, is now replaced by a pathological phase comprised of "either-or," ritualistic repetitive acts, —a caricature of order-, with entrainments of ideas such as obsessions. There is also a notable difficulty in bringing closure to a thought as well as thoughts that are intrusive, rebellious, and "nasty" quickly alternating with piety and/or periods of slovenlyness or self-neglect [41].

The existence of an overall operating mode of the brain's neural network is mentioned explicitly in the literature $[21,42]$. This overall mode normally imparts and then oversees the synchrony, coordinated smoothness, and amalgamated subtlety exhibited during the expression of all individual higher faculties. It is similar to the functions mediated by the basal ganglia that insure the coordinated smoothness and elegance exhibited during our body movements, providing the typical, non-robotic appearance of humans in motion. It represents the very essence of being normal. The expression of a mental disorder, on the other hand is like the symptomatology of Parkinson's disorder where either-or, spastic, painful-toobserve, zombie-like, movements replace the otherwise normal mode.

\section{A Proposed Natural History and Sequencing in the Development of MMDs}

When all of these phenomena are considered together, we can discern the sequence of events leading to the occurrence of a MMD: individuals with inborn extreme temperamental variances-with the implied underlying extreme structural dendritic variances-make up a group of vulnerable candidates. A certain percentage of these will probabilistically develop MMDs. They all share a common structural vulnerability several steps removed from the final structural/functional reality-i.e., the vulnerability to MMDs may actually originate in the faulty fractal, development of dendrites in the brain that ultimately determine an individual's functional reality.

Whether a disorder will actually develop in a particular area of higher brain function depends upon two primary factors: firstly, the existence of brain's structural variance thus vulnerability in a particular mental faculty, or multiple faculties (e.g., comorbidity) and secondly, the environmental influences that act upon the brain.

A major mental disorder will occur in some of the vulnerable individuals, usually in adolescence, presumebly triggered by hormonal and social pressures as well as by other factors. It is a probabilistic phenomenon amenable to prediction only with statistical methods: about 3\% of the general population worldwide manifests disorders.

If there is an extreme temperamental variance or "com- 
promised" area of the brain controlling mainly mood modulation, an individual will be vulnerable to the future possible occurrence of oscillations resulting mainly in bipolar disorder. If it originates in a functional part of the brain controlling social connectedness, thinking processes and coordination of feelings and ideas, it will be expressed as schizophrenia. If it is expressed in a region of the brain that controls orderliness, sequencing, scheduling, and for-planning, it will be expressed as OCD. Areas of the brain that trigger danger (e.g., anxiety and fear) show up mainly in panic attacks, phobias and anxiety disorders as well as OCDs. If it involves temperamental hyper intensity, it may express itself briefly and periodically, involving some or even all faculties-as in the flaring up of borderline phenomena. If in all faculties then it will result in mixed disorders.

Usually, but not always, the operating mode now becomes unstable and episodically bimodal following the onset of the first occurrence. The victim switches phases periodically from normal to pathological and back again in relapses and remissions. During the periodic appearance of the pathological phase, the particular faculty/function affected expresses itself in an either-or fashion with the appearance of symptoms as clusters of oscillating, antithetical substitutes characteristic of each disorder. It is as if the conductor of an orchestra during a musical performance suddenly abandons the orchestra. These phenomena are akin to Parkinson's disease, where awkward, spastic, "either-or" and "zombie-like," painful-to-observe body movements are replaced by normal behavior. The later symptoms are clearly mediated by physical defects in the basal ganglia unable to maintain the synchrony and elegance of the body in motion.

Stemming from these considerations, the statistical prevalence for each MMD can be construed as a downside tradeoff of the brain evolutionarily deploying its advantages. The preexistence of temperamental extreme variances for all MMDs suggests a common underlying vulnerability, with the implied underlying structural variances antedating the psychotic phase of the disorders.

\section{Conclusions}

Consideration of the collective significance of the shared clinical phenomena discussed above suggests novel ways of answering baffling questions about "how-does-thebrain-does-it”, both in health and disease alike. In this paper, some propositions have been made as to their significance, based on the persuasiveness of the readily observable phenomena. Becoming alert to these will protect against reaching erroneous conclusions in clinical studies due to rigid Procrustean guidelines for selection of subjects. Our proposed concepts also promote new ways of viewing the mechanisms underlying the development of mental disorders and will hopefully promote development of new, more effective and safer therapies. An interesting example may be the use of modulated chaotic low-amplitude electric signals applied in lieu of ECT in an effort to restore the brain's normal operating mode, albeit easily and without the problems associated with it.

To close with a metaphor: "In busily studying an individual tree, there is merit in pausing and considering the forest as a whole, with its own emergent qualities.”

\section{Acknowledgements}

My gratitude and appreciation go to Dr. Nicholas Stratas, MD, DLFAPA, Dr. David Servan-Schreiber, MD, PRD, Dr. Robert Wehbie, MD, Ph.D., Dr. Peter Pediaditakis, Ph.D., and Dr. John Rather, Ph.D. for helping and advising me as to how to best express my concepts in this paper; and also to my daughter for editing it.

\section{REFERENCES}

[1] S. Chess and A. T. Chess, "Temperament: Theory and Practice,” Brunner/Mazel, New York, 1996.

[2] N. Pediaditakis, "Borderline Phenomenon Revisited: A Synthesis,” Psychiatric Times, 2002, pp. 37-41.

[3] H. J. Eysenck, "The Definition and Measurement of Psychotism,” Personality and Individual Differences, Vol. 13, No. 7, 1992, pp. 757-785. doi:10.1016/0191-8869(92)90050-Y

[4] C. G. Jung, "Psychological Type," Princeton University Press, Harcourt \& Brace, Princeton, 1971.

[5] C. R. Cloninger, D. M. Svrakic and T. R. Przybeck, "A Psychobiological Model of Temperament and Character," Archieves of General Psychiatry, Vol. 50, No. 12, 1993, pp. 975-990. doi:10.1001/archpsyc.1993.01820240059008

[6] K. David and M. Bates, "Please Understand Me: Character \& Temperament Types,” Prometheus Nemesis Book Co., Carlsbad, 1998.

[7] N. Pediaditakis, "Shared Characteristics in the Clinical Expression and Pharmacological Responses of Mental Disorders and Their Possible Collective Significance," Medical Hypotheses, Vol. 50, No. 4, 1998, pp. 347-352. doi:10.1016/S0306-9877(98)90124-4

[8] N. C. Andreasen, "Creativity and Mental Illness Prevalence Rates in Whites and Their First Degree Relatives," American Journal of Psychiatry, Vol. 144, No. 9, 1987, pp. 1288-1292.

[9] K. R. Jamison, "Mood Disorders and Patterns of Creativity in British Writers and Artists," Psychiatry, Vol. 32, 1989, pp. 125-134.

[10] K. R. Jamison, "Touched by Fire: Manic-Depressive Illness and the Artistic Temperament,” The Free Press, New York, 1993.

[11] J. E. Steinmetz, "The Study of Temperament: Changes, Continuities, and Challenges,” Lawrence Erlbaum Associates, Hillsdale, 1986. 
[12] S. Arieti, "Premorbid Personality in Schizophrenia," American Handbook of Psychiatry, NY Basic Books, New York, 1959, p. 472.

[13] A. R. Yung and P. D. McGorry, "The Prodromal Phase of First Episode Psychosis: Past and Current Conceptualizations,” Schizophrenia Bulletin, Vol. 22, No.2, 1996, pp. 353-370. doi:10.1093/schbul/22.2.353

[14] H. I. Kaplan, B. J. Sadock and J. A. Grebb, "Positive and Negative Symptoms of Schizophrenia," Synopsis of Psychiatry, 7th Edition, Williams \& Wilkins, New York, 1994.

[15] D. R. Hirshfeld-Becker, "Behavioral Inhibition and Disinhibition as Hypothesized Precursors to Psychopathology: Implications for Pediatric Bipolar Disorder,” Biological Psychiatry, Vol. 53, No. 11, 2003, pp. 985-999. doi:10.1016/S0006-3223(03)00316-0

[16] A. M. Benis and J. H. Rand, "A Model of Human Personality Based on Mendelian Genetics," Proceeding of American Association for the Advancement of Science, Vol. 86, No. 5, 1986, p. 124.

[17] A. Szöke, F. Schürhoff, F. Bellivier, F. Rouillon and M. Leboyer, "Temperament in Schizophrenia: A Study of the Tridimensional Personality Questionnaire (TPQ),” European Psychiatry, Vol. 17, No. 7, 2002, pp. 379-383. doi:10.1016/S0924-9338(02)00700-9

[18] American Psychiatric Association, "Diagnostic and Statistical Manual of Mental Disorder," 4th Edition, Washington DC, 1994.

[19] V. Arolt and H. Dilling, “Confounding Diagnostic Systems: A Major Risk in the Use of Criteria-Based Manuals,” Psychopathology, Vol. 27, No. 1-2, 1994, pp. 58-63. doi:10.1159/000284849

[20] J. A. Neal and R. J. Edelmann, "The Etiology of Social Phobia toward a Developmental Profile,” Clinical Psychological Review, Vol. 23, No. 6, 2003, pp. 761-786.

[21] N. Pediaditakis, "Deterministic Nonlinear Chaos in Brain Function and Borderline Psychopathological Phenomena," Medical Hypotheses, Vol. 39, No. 1, 1992, pp. 6772. doi:10.1016/0306-9877(92)90143-Z

[22] H. M. Maru, D. M. Kathuku and D. M. Ndetei, "Psychiatric Morbidity Among Children and Young Persons Appearing in the Nairobi Juvenile Court, Kenya," East African Medical Journal, Vol. 80, No. 6, 2003, pp. 226-232.

[23] F. G. Moeller, E. S. Barratt, D. M. Dougherty, J. M. Schmitz and A. C. Swann, "Psychiatric Aspects of Impulsivity,” American Journal of Psychiatry, Vol. 158, No. 11, 2001, pp. 1783-1793. doi:10.1176/appi.ajp.158.11.1783

[24] C. Henry, "Affective Instability and Impulsivity in Borderline Personality and Bipolar II Disorders, Similarities and Differences,” Journal of Psychiatr Research, Vol. 35, Vol. 6, 2001, pp. 307-312.

[25] D. N. Klein and J. E. Schwartz, "The Relation between Depressive Symptoms and Borderline Personality Disorder Features over Time in Dysthymic Disorder,” Journal of Personal Disorder, Vol. 16, No. 6, 2002, pp. 523-535. doi:10.1521/pedi.16.6.523.22143

[26] G. Claridge, “Creativity and Madness: Clues from Mod- ern Psychiatric Diagnosis,” Genius and the Mind, Oxford University Press, Oxford, 1980.

[27] K.-H. Reger and H. Dilling, "History of Psychiatry in Lubeck: The Nineteenth Century," History of Psychiatry, Vol. 5, No. 18, 1994, pp. 157-174. doi:10.1177/0957154X9400501801

[28] M. A. Taylor, S. A. Berenbaum, V. C. Jampala and C. R. Cloninger, "Are Schizophrenia and Affective Disorder Related? Preliminary Data from a Family Study,” American Journal of Psychiatry, Vol. 150, Vol. 2, 1993, pp. 278-285.

[29] D. M. Fergusson, L. J. Horwood and M. T. Lynskey, "Prevalence and Comorbidity of DSM-III-R Diagnoses in a Birth Cohort of 15 Year Olds," Journal of the American Academy of Child, Vol. 32, Vol. 6, 1993, pp. 1127-1134.

[30] A. E. Doyle and S. V. Faraone, "Familial Links between Attention Deficit Hyperactivity Disorder, Conduct Disorder and Bipolar Disorder," Current Psychiatry Reports, Vol. 4, 2002, pp. 146-152. doi:10.1007/s11920-002-0049-y

[31] B. F. Grant, "Prevalence, Correlates, and Disability of Personality Disorders in the United States: Results from the National Epidemiologic Survey on Alcohol and Related Conditions,” Journal of Clinical Psychiatry, Vol. 65, 2004, pp. 948-958. doi:10.4088/JCP.v65n0711

[32] N. Pediaditakis, "The Occurrence of Schizophrenia in Monozygotic Twins and Fractal, Dentritic Development," Archives of General Psychiatry, Vol. 53, No. 1, 1996, p. 85. doi:10.1001/archpsyc.1996.01830010087014

[33] W. F. Baare, et al., "Volumes of Brain Structures in Twins Discordant for Schizophrenia," Archives of General Psychiatry, Vol. 58, No. 1, 2001, pp. 33-40. doi:10.1001/archpsyc.58.1.33

[34] M. P. Freeman, S. A. Freeman and S. L. McElroy, "The Comorbidity of Bipolar and Anxiety Disorders: Prevalence, Psychobiology and Treatment Issues," Journal of Affect Disorder, Vol. 68, No. 1, 2002, pp. 1-23. doi:10.1016/S0165-0327(00)00299-8

[35] R. McIntyre and M. Katzman, "The Role of Atypical Antipsychotics in Bipolar Depression and Anxiety Disorders,” Bipolar Disorder, Vol. 5, Suppl. s2, 2003, pp. 20-35.

[36] H. Akiskal, "Temperament: The Bridge between Biology and Affective Illness,” Annals of General Psychiatry, Vol. 2, Suppl. 1, 2003, p. 548.

[37] L. N. Yatham, "Efficacy of Atypical Antipsychotics in Mood Disorders," Journal of Clinical Psychopharmacology, Vol. 23, No. 3, 2003, pp. 9-14. doi:10.1097/01.jcp.0000084036.22282.ea

[38] A. Breier, "Effects of Clozapine on Positive and Negative Symptoms in Outpatients with Schizophrenia," American Journal of Psychiatry, Vol. 151, No. 1, 1994, pp. 20-26.

[39] R. F. Asarnow, "Schizophrenia and Schizophrenia-Spectrum Personality Disorders in the First-Degree Relatives of Children with Schizophrenia," Archives General Psychiatry, Vol. 58, No. 6, 2001, pp. 581-588. doi:10.1001/archpsyc.58.6.581

[40] C. Wijeratne, G. S. Halliday and R. W. Lyndon, "The 
Present Status of Electroconvulsive Therapy: A Systematic Review," Medical Journal of Australia, Vol. 171, No. 5, 1999, pp. 250-254.

[41] C. A. Skarda and W. J. Freeman, "How Brains Make Chaos in Order to Make Sense of the World,” Behavioral and Brain Sciences, Vol. 10, No. 2, 1987, pp. 161-195. doi:10.1017/S0140525X00047336

[42] C. King, "Fractal and Chaotic Dynamics in Nervous Systems,” Progress in Neurobiology, Vol. 36, No. 4, 1991, pp. 279-308. doi:10.1016/0301-0082(91)90003-J 